\title{
Comparative Research of Magnetic Bead and Traditional Methods to Extract the Genomic DNA of Bean Sprouts
}

\author{
Yanwei Cheng, Shengnan Kong, Huiyun Li, Shuang \\ Han, Jianming Han*, Xueyi Yang \\ College of Life Sciences ,Luoyang Normal University, \\ Luoyang, China \\ 471022 \\ e-mail: hjm@lynu.edu.cn
}

\author{
Lihui Xiao, Deguang Du \\ Henan Huier Nano Technology Co., Ltd. \\ Luoyang, China \\ 471000
}

\begin{abstract}
The Young leaves of green beans sprouts were used as experimental materials, total DNA was extracted by using methods including magnetic bead and traditional SDS methods. Integrality and purity of nucleic acids were detected with agarose gel electrophoresis, ultraviolet absorption and PCR.The results showed that: the total DNA of green beans sprouts extracted by using magnetic beads method had the advantages of high yield and high quality, agarose gel electrophoresis bands is clear and complete, meeting the requirements of the follow-up molecular biology experiments. the total DNA extracted by using SDS method has the characteristics of low production, impurities, easily degradable, agarose gel electrophoresis bands is not clear and complete. Experiments also demonstrated: Compared with SDS method, magnetic beads method to extract DNA had the advantages of simple operation, short time-consuming, stable, high purity, more conducive to rapid DNA detection.
\end{abstract}

Keywords: magnetic bead method; DNA ; SDS method;Young leaves of bean sprouts

The high quality of DNA and RNA sample is the foundation of molecular biology, in the plant nucleic acids extraction process(including DNA and RNA), the ability to effectively remove the material polysaccharides, lipids and polyphenols, and inhibition of DNA and RNA enzyme activity. It is an important factor of the extraction of nucleic acid and the quality of nucleic acid about samples ${ }^{[1-2]}$. You can select the appropriate extraction methods from the feature and test requirements of different plant materials in practical work ${ }^{[3]}$.

There are many DNA extraction methods used at home and abroad, in this paper, we choose the lasted magnetic bead method and traditional laws SDS method ${ }^{[4]}$ to extract the genomic DNA of young leaves of sprouts. The purpose of this paper is to compare the two methods of DNA extraction, so to verify all the advantages of magnetic bead method, and makes high-purity DNA extraction experiments can be conducted in students's biology, genetics laboratory of college students and middle school.

In order to explore the object of young leaves of green sprouts, we use the magnetic bead kit to extract genomic DNA, then after extraction of the DNA solution, we get on trace nucleic acid analyzer measurement and agarose gel electrophoresis, verified and analyzed the effect of magnetic bead extraction ${ }^{[5]}$.
The principle of magnetic beads method is to destruction of animal and plant cells by using the cell lysate containing the protein denaturation agent, and to make the DNA and protein separation, thus free release; magnetic beads can specifically adsorb nucleic acid, through washing, removing impurities such as protein, polysaccharide except for nucleic acid ; because the eluate can dissociation of DNA on magnetic beads, high purity and concentration of DNA can be obtained and used for PCR template, genetic engineering, gene chip technology ${ }^{[6]}$, etc.

The principle of SDS method: SDS is a kind of anionic detergent which can dissolve the cell membrane and nuclear membrane protein, depolymerize the nuclear protein so that DNA can be free, then remove the protein through chloroformextracting, the remaining DNA with anhydrous ethanol precipitation ${ }^{[7]}$.

\section{EXPERIMENTAL MATERIALS AND EQUIPMENT}

\subsection{Experimental Materials and Reagents}

Mung bean sprouts (commercially available); Henan Hui Er Nami biotechnology company produced magnetic beads plant genomic DNA extraction kit; Cyanine dye; Agarose, bromophenol blue, Tris were purchased from Biological Engineering (Shanghai) Co., Ltd, TE buffer; Ultra-pure water, DNA extract, chloroform, ethanol, 70\% $(\mathrm{V} / \mathrm{V})$;

\subsection{Laboratory instruments}

DYY-8C type electrophoresis apparatus of Beijing sixty-one Instrument; Q5000 Quawell UV VIS spectrophotometer for the determination of trace nucleic acid; Galanz microwave oven; The SVC-2000 high-precision automatic exchange regulator power supply from Shanghai People Enterprise (group) Co., Ltd; The Tocan-320 gel imaging system from Shanghai Lingcheng biotechnology Ltd.; Computer; Electric heated water bath from Beijing Yong Guangming Medical Instrument Factory; TGL-16G centrifuge of Shanghai Anting scientific instrument factory; HRCLJ-02-type tin magnetic separator from Henan Hui Er Nami Technology, Inc.; FA-1004 electronic balance from Shanghai Shangtian Precision Instrument Co., Ltd.; XK98-A electronic quartz timer from Xinkang Medical Devices Co., Ltd.; Xinfei 
refrigerator; pipette; 100ml cylinder; flasks; $1.5 \mathrm{mlEP}$ tube; 200ulPCR tube; mortar.

\section{DNA EXTRACTION AND ANALYSIS}

\subsection{Sampling}

Using electronic balance to weigh part of mung bean sprouts leaves in the weighing paper as shown in table 1 , temporary preservation in the shadows.

TABLE 1 WEIGH PART OF MUNG BEAN SPROUTS LEAVES

\begin{tabular}{|c|c|c|}
\hline & $\begin{array}{c}\text { magnetic bead } \\
\text { method(mg) }\end{array}$ & SDS(g) \\
\hline Sample 1-1 & 17.1 & \multirow{2}{*}{0.3025} \\
\hline Sample 1-2 & 17.5 & \\
\hline Sample 1-3 & 17.6 & \multirow{2}{*}{0.3036} \\
\hline Sample 2-1 & 33.6 & \\
\hline Sample 2-2 & 34.1 & \multirow{2}{*}{0.3026} \\
\cline { 1 - 2 } Sa & 33.4 & \\
\hline mple 2-3 Sample 3-1 & 52.3 & \\
\hline Sample 3-2 & 52.0 & \\
\hline Sample 3-3 & 52.6 & \\
\hline
\end{tabular}

\subsection{Using magnetic bead method to extract DNA.}

Cracking:Put the weighed sample into a mortar grinding adding 400ul BufferA, mix transferred to $1.5 \mathrm{ml}$ EP tube. Then add DTT10ul, protease -K5ul, then place the EP tube in a thermostatic water tank $65^{\circ} \mathrm{C}$ incubated for 20min, every $2 \sim 3 \mathrm{~min}$ jiggle EP tube mixing, and then take out the EP tube, 12000r centrifugal $5 \mathrm{~min}$.

Binding: Draw supernatant 300ul to new $1.5 \mathrm{ml}$ EP tube from the tube after centrifugation, join 3000 ul oscillation mixes uniform magnetic beads with liquid, turbulence mixing, place at room temperature for $5 \mathrm{~min}$ (If stratification, then slightly shaking uniform), get on magnetic separation, with a smaller tip aspirating waste and avoid absorb magnetic beads.

Washing: Join 500ul wash solution I , shaken gently mixing, placing $10 \mathrm{~S}$, then put the EP tube in magnetic frame 30S, magnetic separation, after the beads attracted to the wall, aspirating waste. Then add 500ul wash solution II , repeat the above operation, after washing two times of washing liquid II, thoroughly clean the residual liquid of the net tube cover and the bottom of the tube, open the cover at room temperature and dry the $2 \mathrm{~min}$.

Eluting: Join 100ul eluent, slowly sucking mix, $65^{\circ} \mathrm{C}$ water bath 10min. Every $2 \sim 3 \mathrm{~min}$ jog, mix EP tube. Then magnetic separation, carefully draw the supernatant (ie DNA solution) to the new EP tube.

Identification of DNA: 10ul DNA solution was taken for electrophoresis, and the DNA bands were observed after $20 \mathrm{~min}$.

\subsection{Using SDS method to extract DNA ${ }^{[8]}$}

(1)The sample is placed in pre cooling of the mortar in grinding, as thin as possible, then transferred to $1.5 \mathrm{ml} \mathrm{Ep}$ tube, turn over $600 \mathrm{ul}$ extract, $65^{\circ} \mathrm{C}$ water bath $10 \mathrm{~min}$.

(2)Centrifuged $10 \mathrm{~min}$ in $8000 \mathrm{r} / \mathrm{min}$, then take the supernatant.

(3)Adding 600ul of chloroform, shocking, then centrifuged $5 \mathrm{~min}$ in $13000 \mathrm{r} / \mathrm{min}$.
(4)Take the supernatant and add 2 times volume of pre cooling absolute ethanol, gently mix by inversion.

(5)Then centrifuged $10 \mathrm{~min}$ in $13000 \mathrm{r} / \mathrm{min}$.

(6)Discard supernatant, the precipitation was washed twice with 70\% (V/V)ethanol. Dried at room temperature.

(7)The precipitate was dissolved in 30ul sterile double distilled water.

(8)The 10ul DNA solution was taken for electrophoresis, viewed in the ultraviolet observation instrument after $20 \mathrm{~min}$, the proposed DNA bands.

\section{DETECTION METHOD}

\subsection{The detection of DNA concentration, purity and absorbance}

30min advance open the trace nucleic acid analyzer preheating, followed by ultrapure water trace nucleic acid analyzer once, then draw 3ul eluate as blank control, measurement, and to ensure the absolute value 260Abs and $280 \mathrm{Abs}$ of less than 0.04 , 3ul DNA solution was then added to the trace nucleic acid analyzer, performed trace nucleic acid analyzer measurement, after the measurement, save the results, later analysis.

\subsection{Identification of agarose gel electrophoresis}

\subsubsection{With glue}

Weigh $0.40 \mathrm{~g}$ agarose powder poured into the flask, mixed and heated in the microwave for 1 to 2 minutes until the agarose is completely melted, pour inserted comb offset, two hours later, pulling the comb, waiting for the sample.

\subsubsection{Indicator solution spotting}

Remove 12 200ul PCR tube, followed by marks from 1 to 12 , and added bromine phenol blue solution $2 \mathrm{ul}$ and fluorescent dye 1.5ul.

\subsubsection{DNA sample}

Carefully draw the DNA solution 10ul in turn, carefully added to the 200ul PCR tube, slowly pumping twice, mixing, accurately injected into the holes on the rubber block, each hole represents a lane.

\subsubsection{Electrophoresis}

Gently put the rubber block fitted into electrophoresis buffer electrophoresis tank in the middle of the pallet (Note: don't let dye ran out of the sample hole), one end of the sample hole toward the negative power supply section, placed after inspection to ensure that the electrode buffer full spotting holes. Open the power switch of the electrophoresis instrument, set the control voltage of $120 \mathrm{~V}$, the electrophoretic time of $30 \mathrm{~min}$, and run the glue. 
IV. EXPERIMENTAL RESULTS AND ANALYSIS

4.1 Detection results and analysis of the trace nucleic acid nucleic acid detector for bean sprouts genomic DNA

TABLE 2 THE EXTRACTION OF GENOMIC DNA MAGNETIC BEADS METHOD RESULTS OF BEAN SPROUTS

\begin{tabular}{cccccccc}
\hline No. & SW (nm) & SW Abs & $\begin{array}{c}260 \mathrm{Abs} \\
10 \mathrm{~mm}\end{array}$ & $\begin{array}{c}280 \mathrm{Abs} \\
10 \mathrm{~mm}\end{array}$ & $260 / 280$ & $260 / 230$ & $\begin{array}{c}\text { Conc. } \\
(\mathrm{Ng} / \mathrm{ul})\end{array}$ \\
\hline Blank & 320 & 0 & 0.001 & -0.001 & -1.19 & -0.53 & 0.1 \\
$1-1$ & 320 & 0.06 & 11.074 & 5.565 & 1.99 & 2.02 & 553.7 \\
$1-2$ & 320 & 0.084 & 12.645 & 6.382 & 1.98 & 1.96 & 632.3 \\
$1-3$ & 320 & 0.031 & 11.287 & 5.651 & 2 & 1.92 & 564.3 \\
$2-1$ & 320 & 0.081 & 20.44 & 10.163 & 2.01 & 1.95 & 1022 \\
$2-2$ & 320 & 0.109 & 27.308 & 13.528 & 2.02 & 2.01 & 1365.4 \\
$2-3$ & 320 & 0.078 & 25.746 & 12.703 & 2.03 & 2.03 & 1287.3 \\
$3-1$ & 320 & 0.152 & 35.028 & 17.312 & 2.02 & 1.93 & 1751.4 \\
$3-2$ & 320 & 0.136 & 30.373 & 15.075 & 2.01 & 1.93 & 1518.7 \\
$3-3$ & 320 & 0.158 & 31.018 & 15.362 & 2.02 & 1.93 & 1550.9 \\
\hline
\end{tabular}

\subsubsection{Detection results of trace nucleic acid detector}

The conjugated double bonds, in the purine and the pyridine of nucleic acids, have a strong absorption to the ultraviolet. In the UV region, DNA'slambda max is $260 \mathrm{~nm}$, protein is $280 \mathrm{~nm}$, phenols and small molecule salt is 230nm. The A260/A280 value of pure double stranded DNA should be near 1.8. Which is below 2.8 indicate DNA contains small molecular impurities such as proteins and amino acids, phenols and so on, and which is above 2.8 indicate DNA contains RNA. If the A260/A230 value more than 2.0 shows that the phenol and the salt salt remove very cleanly ${ }^{[9]}$.

The beads extraction DNA measurement results are shown in Table 2:(1) Sprouts A260 / A280 at 1.98 2.03, with an average of 2.01, indicating that the extracted DNA
UV absorption curve has a typical natural DNA standard absorption spectroscopy, protein removal better. Since the experiment did not add RNase, so the results contained in the RNA. (2) Processing bean sprouts A260 / A230 at 1.92 $\sim 2.03$, with an average of 1.96, indicating that the removal of phenol and small molecular compounds was complete. (3) DAN bean yield and quantity of samples is basically linear, indicating magnetic beads extraction kit are stable.

SDS method to extract DNA, because of sample consumption is much more than the magnetic beads, so don't use trace nucleic acid detector to test, so there is no use trace nucleic acid detector test, identification results from agarose gel electrophoresis may also be obvious effect on the extraction of SDS method.

4.2 DNA identification results and analysis of agarose gel electrophoresis

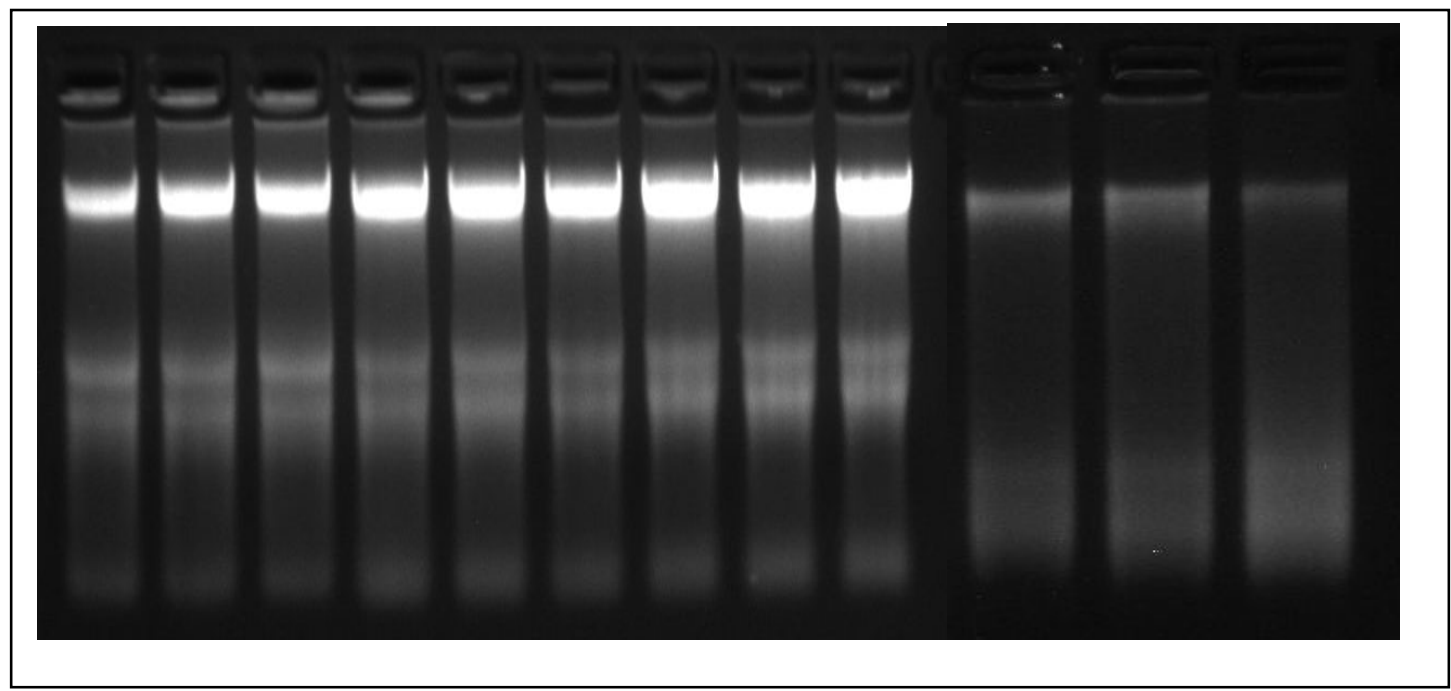

Figure 1 bean sprouts genomic DNA electrophoresis

From left to right 1 to 3 lanes for immunomagnetic 1DNA bands extracted samples; 4 to 6 lane for samples of 2DNA strip magnetic beads method; 7 to 9 lane for samples of 3DNA strip magnetic beads method;the last three lanes for SDS extraction of DNA bands. 
After electrophoresis, turn off the power, gently remove the rubber block (along with the glue frame), then, take the rubber block into nucleic acid gel imaging system, observed under 320nm UV, and photographed electrophoresis, as shown in Fig.1.

experimental material in the bean sprouts leaves, the electrophoresis bands were linear, no tail, with a clear pattern formation, less protein contamination, no DNA degradation phenomenon, by contrast, more sample strips slightly less bright than the sample. But the quantity of sample using SDS method is several times or even ten times of the magnetic bead method, but extraction young leaves of sprouts DNA bands is less bright than beads method, visibling SDS method consumes too much material, extraction DNA content and extraction process of DNA degradation, and contains many impurities, with no clear magnetic bead method.

\section{V.DISCUSSION}

The experiment proved that the method of magnetic beads to extract DNA had greater advantages such as high efficiency, convenience, security, stability, high reliability, compared with the traditional method of SDS.

SDS method is cumbersome, many supplies, time-consuming, low efficiency, DNA impurities extraction, but also involves many harmful reagents, such as chloroform, etc., contrary to low-carbon environmental protection concept. With magnetic beads method extract DNA research in-depth and popularization and application, promote the development of domestic basic biology, at present bead method extraction DNA in economically developed countries has been applied to bulk, without a lot of consumables, not in contact with phenol / chloroform and other toxic reagent, the operation is simple and convenient, separation and purification of DNA only 30 min. Electrophoresis simply for $30 \mathrm{~min}$, can be observed as bright and clear DNA bands, and high purity of the extracted DNA, complete segments, can be directly used for subsequent PCR amplification, and is easy to implement automated operation, accord with high standard and high efficiency of the modernization of education and sustainable development concept, with timely and advanced.
Identified DNA by agarose electrophoresis is a common method for the scientific identification of university and laboratories. As shown in Figure 1, after a $1 \%$ agarose gel electrophoresis detection, using magnetic beads method to extract high quality DNA from the

A fast, economical, safe and efficient method for the extraction of animal and plant genome will be the hope of many researchers research on biological macromolecules in the body and their application rule along with the continuous development of biotechnology. If the magnetic beads method is applied to hot research in plant (such as pepper) and animal (such as human mites, aphids) genome extraction, , it will greatly accelerate the rapid development of cell biology.

\section{ACKNOWLEDGEMENT}

This work was supported by the NSFC-Henan province joint foundation (U1204307), the Natural Science Foundation of Henan Province ( No.2010B180020)

\section{REFERENCES}

[1] Zhang Zhigang,Li Xun,GuanQingyun etc.. The SDS method of improved extraction of rice endosperm and Arabidopsis Style DNA and RNA. Plant Physiology Communications, 2006(3):493-495.

[2] Chen Honglin, Wang Xi, Hezhen Yan. An effective method for extraction of high quality of mangrove plant RNA. Chinese Journal of Biological Engineering, 2010,30( 6) : 84-88.

[3] Party Wei, Wei Ya-Hui, Zhang Huaping, etc.. Comparison of total DNA extraction methods from grape[J]. Journal of Northwest A\&F University. Natural Science, 2003.33(5):572-574.

[4] Li Jidong, Liang Qiwei, Wu Wenjuan Mao Baiyang. A comparative study on genomic DNA extraction of mature leaves[J]. Henan Science,2009,27,(3): 285-288.

[5] Cui Yali, Li Zheng, Fang Yu. The magnetic particles used in nucleic acid research. Journal of Northwest A\&F University,2000,28(1): 80-85.

[6] Yuan Wenlong,Kang Zhanhai,Tao Bu, etc.. Gene chip technology and its application in plants. Anhui Agricultural Science,2010,38(26): 14279-14280,14297.

[7] Guo Hongyuan, She Maoyun. An Improved Total DNA Extraction Method[J]. Shanxi Agricultural Science,2009,37,(2):3-5.

[8] Liu Jian. Molecular biology and genetic engineering experiments Tutorials. Beijing: Science Press,2008.

[9] Wang Yan,Zhang Baoxi,Zhang Guoyu,etc.. Study on the optimization of DNA extraction method for pepper leaves. Yangtze vegetables newspaper,2006.08. 